REFERENTES NACIONALES DEL CONCEPTO DE AUDITORÍA EN SALUD

CARMEN MARÍA VARGASMONTAÑA

FISIOTERAPEUTA

ERIKA LORIETH VARGAS MONTAÑA

BACTERIOLOGA

MONOGRAFÍA PARA OPTAR AL TÍTULO DE AUDITORA DE SALUD

UNIVERSIDAD SANTO TOMÁS

FACULTA DE ECONOMÍA

ESPECIALIZACIÓN EN AUDITORÍA EN SALUD

SEDE BOGOTÁ

2018 
CARMEN MARÍA VARGASMONTAÑA

ERIKA LORIETH VARGAS MONTAÑA

ASESORA:

ALEJANDRA VALENZUELA CAZÉS

MAGISTER EN SALUD PÚBLICA

UNIVERSIDAD SANTO TOMÁS

FACULTA DE ECONOMÍA

ESPECIALIZACIÓN EN AUDITORÍA EN SALUD

SEDE BOGOTÁ

2018 
TABLA DE CONTENIDO

1. PROBLEMA DE INVESTIGACIÓN

2. OBJETIVOS

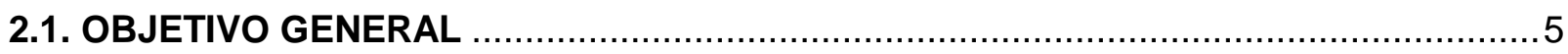

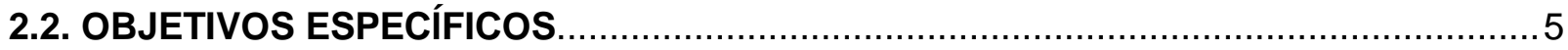

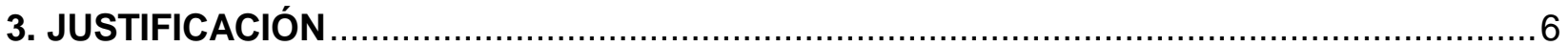

4. ELEMENTOS CONCEPTUALES

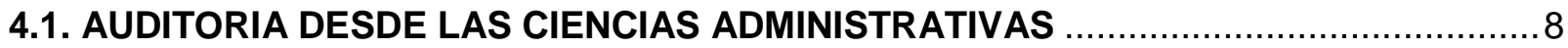

4.2. PILARES DE LA CALIDAD

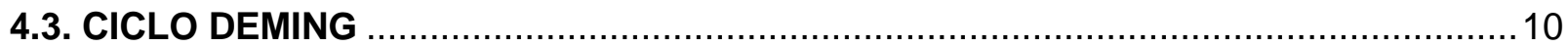

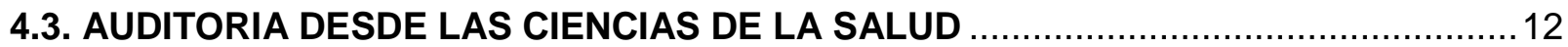

5. METODOGIA

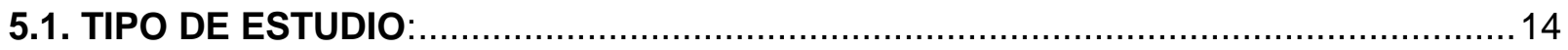

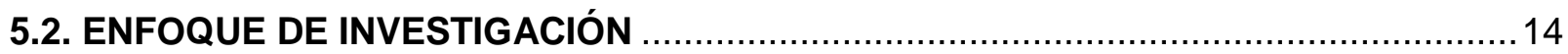

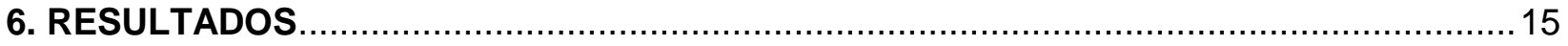

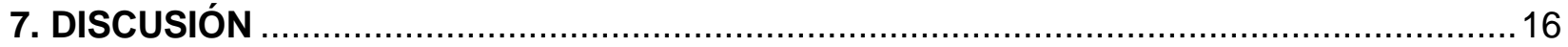

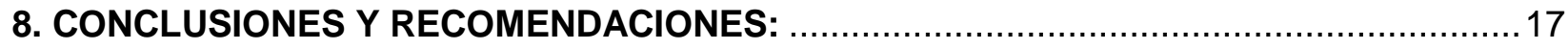

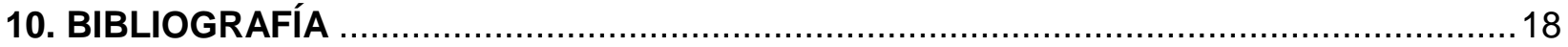




\section{PROBLEMA DE INVESTIGACIÓN}

En el presente apartado se desarrolla la siguiente pregunta de investigación: ¿Que sería del sistema de salud sin el papel del auditor de salud?

Acerca del sistema de salud en Colombia en sus estrategias, principios y medidas ha propiciado situaciones conflictivas entre los diferentes actores del Sistema, que dificultan el funcionamiento del Sistema en detrimento de la protección y garantía servicio a la salud, dentro de estas situaciones, se encuentran principalmente: la falta de control del Estado, la prevalencia de los intereses privados sobre el derecho fundamental a la salud, la intermediación de las EPS y la existencia del POS. (1)

En cuanto a esa falla de la vigilancia y control por parte del Estado, hay quienes consideran que los problemas de calidad tienen que ver esencialmente con la escaza supervisión y vigilancia que ejerce la Superintendencia Nacional de Salud sobre las EPS. Estas entidades han creado carteles de la salud para manipular el Sistema a su conveniencia, y poco o nada ha hecho el Estado para enfrentar esta situación. (1)

Como ejemplo de esto podemos hablar de la experiencia de la empresa prestadora de servicios Saludos quien, en sus 10 años de denuncias y dudas en sus acciones, solo hasta mayo de 2011 se tomó la decisión de intervenirla, a razón de una auditoria exhaustiva. En ésta se encontraron inconsistencias en su contabilidad, principalmente entre las deudas reales y los recobros hechos al FOSYGA con lo que al parecer financiaron su crecimiento desmedido y la compra de bienes raíces ajenos a la naturaleza jurídica de la Entidad. (2) 
Teniendo en cuenta entonces éstas situaciones conflictivas, se hace necesaria la prevalencia de las auditorias y en esa misma medida del papel del auditor, en el cual ellos como actores fundamentales procedan a evaluar las actividades, funciones y procedimientos de los servicios prestados por las instituciones de salud y su interés en la calidad de la atención en salud.

\section{OBJETIVOS}

\subsection{OBJETIVO GENERAL}

Analizar las perspectivas teóricas acerca de los referentes nacionales del concepto de auditoría con el fin de dar soporte teórico para el mejoramiento de la atención y prestación en los servicios de salud.

\subsection{OBJETIVOS ESPECÍFICOS}

- Especificar las perspectivas teóricas partiendo desde los referentes nacionales de la auditoria en salud por medio de una revisión bibliográfica sustentada.

- Justificar la importancia de la auditoría de salud como un quehacer profesional especializado, el cual es preponderante para el mejoramiento y optimización de los servicios en salud con eficacia, eficiencia y efectividad. 


\section{JUSTIFICACIÓN}

Una auditoria en su etimología y significado conceptual se define como una evaluación que busca identificar errores dentro de un contexto o una serie de procesos. Esta percepción puede limitar, de alguna manera, los alcances de la auditoría como una herramienta útil en el mejoramiento continuo de la calidad de los servicios en salud. Por lo tanto, se hace necesario definir la auditoría desde un punto de vista integral en el que se incluyan los términos de calidad de los servicios, oportunidad, acceso y humanización. De igual forma, al comprender la auditoría en salud como una herramienta que permite la identificación de las falencias en la prestación de los servicios se mitigaría el impacto que esta puede generar sobre los usuarios, ya que al identificar una falla en los procesos se obtendría una oportunidad para solventarlo, lo que conllevaría al mejoramiento continuo y, por ende, a la calidad del proceso o servicio (3).

Por esta razones, el auditor debe tener presente una serie de conocimientos teóricos planteados por Donadebian (3) y el Ciclo PHVA que en su quehacer profesional le permite conocer los aspectos más relevantes de lo que implica la auditoría en salud permitiría desarrollar estrategias encaminadas a la identificación de oportunidades de mejoramiento continuo dentro de las instituciones, estos pilares del Ciclo Phva son los siguientes:

- Planear $(\mathrm{P})$ : Esta fase está compuesta de dos etapas. La primera tiene por objeto identificar metas (qué). La segunda tiene que ver con la definición de los medios (cómo), es decir, las maneras de alcanzar las metas.

- Hacer (H): Esta es la fase de ejecución de los medios establecidos en la fase inicial; a su vez, tiene dos etapas: la primera se orienta a la formación de las personas en las formas o "cómo" establecidos para cumplir la meta; la segunda se presenta formalmente como la ejecución de lo planeado, pero tiene que ver, adicionalmente, con la recolección de los datos.

- Verificar (V): Esta es la fase de verificación de los resultados. Aquí, sobre la base de la evaluación del comportamiento de los indicadores que se han construido o 
de la aplicación de los métodos de evaluación de la calidad, incluidos aquellos que forman parte de la auditoría para el mejoramiento de la calidad, se valida la ejecución de la etapa anterior gracias a los hechos y datos recogidos.

- Actuar (A): En esta cuarta fase es preciso actuar en relación con todo el proceso. Existen básicamente dos posibilidades; en el caso de que la meta haya sido conseguida, es necesario estandarizar la ejecución con el ánimo de mantener los resultados del proceso. (4) 


\section{ELEMENTOS CONCEPTUALES}

\subsection{Auditoria desde las Ciencias Administrativas}

La Auditoria desde la perspectiva Administrativa Comprende el examen del establecimiento y cumplimiento de los planes, políticas, metas y objetivos trazado por la dirección general en todas las frases del proceso administrativo, planeación, organización, dirección, control, evaluación. (5)

Entre los aspectos a examinar podemos señalar:

- Existencia y desarrollo del cumplimiento de la misión, objetivo y entidad de los programas trazados

- Viabilidad de los objetivos trazados

- Conocimiento de meta a corto y largo plazo

- Conformación de una adecuada estructura orgánica

- Selección, contratación, y liquidación del personal

- Existencia y conocimiento de las funciones, manuales, reglamentos, normas legales etc.

- Existencia y aplicación del reglamento interno del trabajo y de seguridad industrial

- Informe oportuno a las identidades estatales

- Oportunidad de la comunicación interna

- Existencia, mantenimiento, conservación, custodia y uso de los bienes

La auditoría desde las Ciencias Administrativas coadyuva con sus análisis a la preparación de la empresa para el futuro, envolver en su análisis los aspectos presupuestales, calidad total y reingeniería en la toma de decisiones. (5)

- APLICACIÓN: Revisar y evaluar si los métodos, sistemas y procedimientos que se siguen en todas las fases del proceso administrativo aseguran el cumplimiento. 
- QUE AUDITA: las políticas, planes, programas, leyes y reglamentaciones que puedan tener un impacto significativo en operación de los reportes

- PARA QUE SE AUDITA: Para asegurar que la organización los esté cumpliendo y respetando los diferentes niveles jerárquicos y operativos, señalando aciertos y desviaciones de aquellas áreas cuyos problemas administrativos detectados exigen una mayor o pronta atención. (5)

\subsection{Pilares de la Calidad}

Desde la perspectiva académica existen un modelo del profesor Adevis Donabedian, quien desarrolló el enfoque de estructura, proceso y resultado para medir y mejorar la calidad de la atención sanitaria.

Dicho modelo se basa en 7 pilares de la calidad que son: eficacia, efectividad, eficiencia, optimización, aceptabilidad, legitimidad, equidad. Cumplir con lo anterior implica un reto para las especializaciones que tienen la misión de evaluar y garantizar la calidad de la asistencia, y además reconocer el significado moral y ético en los contextos sociales, económicos y políticos de un país y, lo más importante, el cuestionarnos de las responsabilidades sociales que repercuten en la atención asistencial legitima y aceptable para el paciente. (6)

Estos pilares se definen cada uno de la siguiente manera:

- Eficacia: es la capacidad de la ciencia y el arte de atención sanitaria para conseguir mejoras en la salud y el bienestar. Significa la mejor actuación posible, en las condiciones más favorables, según el estado del paciente y en unas circunstancias inalterables.

- Efectividad: La efectividad, a diferencia de la eficiencia, es la mejora de salud alcanzada, o que espera conseguir en las circunstancias normales de la práctica 
cotidiana. En la definición y evaluación de la calidad, la efectividad puede especificarse de manera más precisa, como el grado en el que la atención cuya

- Eficiencia: La eficiencia no es más que el coste que representa cualquier mejora en la salud. Si existen dos estrategias de asistencias igualmente eficaces o efectivas, la menos costosa es la más eficiente.

- Optimización. -La optimización se convierte en un tema cuando los efectos de la atención no se valoran en términos absolutos, sino relativos al coste de asistencia.

- Aceptabilidad: Se asume la aceptabilidad como la adaptación de la atención a los deseos, expectativas y a los valores de los pacientes y sus familias. Obviamente los pacientes tienen una expectativa a cerca de los efectos de la asistencia sobre su propia salud y bienestar, y la manera de conseguir estos efectos.

- Legitimidad: En una sociedad democrática, cabe esperar que todas las características de la atención importante para el individuo, sean también asuntos de relevancia social; de eso se trata. No obstante, en un ámbito social, además de la preocupación de los individuos, existe también la preocupación por el bienestar general.

- Equidad: es el principio por el que determina lo que es justo en una distribución de la atención y sus beneficios entre los miembros de una población. La equidad forma parte de aquello que hace que la atención sea aceptable y socialmente legítima para los individuos. (6)

\subsection{Ciclo DEMING}

El Dr. Williams E. Deming es uno de los gurúes de la calidad más conocidos junto con Joseph Juran y Kaoru Ishikawa, entre otros. Con el título de doctorado en física 
matemática obtenido en 1928 trabajó en el US Goverment Service durante varios años en técnicas estadísticas de muestreo. Pero su desempeño toma relevancia después de la segunda guerra mundial cuando, en 1950 fue enviado a Japón en un proyecto de cooperación con Estados Unidos, como consejero del censo de aquel país.(7)

La base conceptual del Ciclo de Deming o P.D.C.A, (los japoneses lo denominaron Ciclo o Ruta de Deming y así se conoce hoy aunque inicialmente fue desarrollado por Shewart), como herramienta fundamental para trabajar sobre la mejora continua está en que las funciones que se cumplen en una institución asistencial, como en cualquier otro tipo de organización estructurado, deben ser planificadas, realizadas y verificadas de modo de tomar las acciones correctivas o preventivas necesarias. (7)

EI Ciclo DEMING comprende los siguientes elementos:

\section{- Planificar}

Es estructurar un proceso sobre la base de un diagnóstico previo. La planificación consta de un análisis de la situación actual o diagnóstico. Luego el establecimiento de principios, metas y objetivos, después la fijación de los medios para lograr los objetivos y por último la Adjudicación de los recursos para gestionar los medios

Por ejemplo, para implantar un sistema de gestión de la calidad, la primera fase de planificación implica el compromiso de la dirección y la segunda parte de programación, entre otras cosas, el diseño del plan.

\section{- Controlar}

El Ciclo Deming no incluye una etapa de Controlar. Controlar generalmente se confunde con el concepto de verificar, aunque son cosas diferentes.

Controlar es verificar y tomar acciones correctivas, es decir, abarca dos de las etapas del Ciclo de Deming: CONTROLAR es VERIFICAR más ACTUAR 


\section{- Hoja de recolección de datos}

Las Hojas de Recolección de Datos, también conocidas como Hoja de Registro, Verificación o Chequeo, son útiles para reunir, cuantificar y clasificar sistemáticamente datos de modo de obtener una imagen clara de los hechos de manera de comenzar a detectar patrones o tendencias $u$ otras informaciones de interés.

La recolección de datos generalmente es el primer paso de casi toda medición estadística y el análisis de los mismos se hará a través de otras herramientas que podrán ser diagramas de barras, diagrama de Pareto, diagramas de dispersión, gráficos de sectores u otro, según el objetivo perseguido.

\section{- Brainstorming o torbellino de ideas}

El Brainstorming, conocido como tormenta o torbellino de ideas es la precursora y seguramente, la más conocida de las técnicas de creatividad.

Partiendo de la base que el activo más valioso de cualquier organización es su gente y la capacidad que tiene para concebir ideas, el Brainstorming se trata de un proceso grupal donde los participantes las generan libremente, sin críticas ni comentarios, lo que constituye una técnica para estimular y fortalecer el proceso del pensamiento creativo.

Conociendo que la mayoría de las personas utiliza solamente el $10 \%$ de su capacidad de reflexión, mediante la creación de grupos y aplicando técnicas de dinámica grupal se cumple con el propósito que es establecer la mayor cantidad de ideas posibles en torno de un problema detectado. (7)

\subsection{Auditoria desde las Ciencias de la Salud}

\section{- Auditoria en General}

Un auditor es un profesional que desarrolla el ejercicio de la auditoria, principalmente en una auditoria se realiza una revisión de la eficiencia, efectividad y utilización de los 
recursos, así como la determinación de si se han logrado los resultados propuestos en una organización, las auditorias pueden ser en salud, médica o clínica las cuales basan su observancia en temas comunes en la rama, en otro sentido la auditoría en salud también llamada auditoria medica está enfocada primordialmente hacia su proceso y resultado que hace parte del sistema obligatoria de garantía de la calidad en salud (6)

\section{- Auditoria en salud}

La auditoría de salud, en el marco del Sistema General de Seguridad Social colombiano, establece a partir de las formulaciones contenidas en el Art. 227 de la Ley 100 de 1993, que define los mecanismos orientadores de la garantía de la calidad. Determina el mismo, el papel del gobierno nacional es expedir las normas que organizan el Sistema Obligatorio de Garantía de Calidad (SOGC), el cual está orientado al objetivo de proveer servicios de salud accesibles y equitativos, teniendo como propositivo la satisfacción de los usuarios. (6)

Por lo tanto, existe una política pública de gestión de la calidad en salud, que desde la perspectiva estatal implica, por extensión, garantizar los derechos fundamentales contemplados en la Constitución Política Nacional, tales como el derecho a la vida y a la salud. El objetivo de garantizar estos derechos es inmanente al quehacer y ejercicio del especialista en auditoría de salud, de ahí que todos los desarrollos normativos formulados en el SOGC son objeto de estudio por parte de los mismos. (7)

El Sistema de Garantía de la Calidad en Salud (SOGC) es el producto más desarrollado de la política pública destinada garantizar la calidad de los servicios de salud. El mismo se sustenta jurídicamente en el Decreto 2309 de 2002 y actualmente se rige por el Decreto 1011 del 2006; también por la Ley 1438 de 2011. El SOGC está conformado por cuatro componentes, a saber: Sistema Único de Habilitación, Auditoría para el Mejoramiento de la Calidad de la Atención en Salud, Sistema Único de Acreditación y Sistema de Información para la Calidad en Salud. Los cuatro componentes sustentan las pautas indicativas de la auditoría para el mejoramiento de la calidad en la prestación de 
los servicios de salud, integrado a normas y políticas de que propendan en el derecho a la salud y humanización de los servicios de salud. (8)

\section{METODOGIA}

\subsection{Tipo de Estudio:}

La presente investigación es de tipo descriptivo, una investigación descriptiva consiste en llegar a conocer las situaciones, costumbres y actitudes predominantes a través de la descripción exacta de las actividades, objetos, procesos y personas. (11)

\subsection{Enfoque de Investigación}

Revisión Bibliográfica con fuentes secundarias y terciarias:

Podemos denominar fuentes primarias a aquél material, que se considera de primera mano, según Silvestrini (2008) las fuentes primarias son aquellas que contienen información original, que ha sido publicada por primera vez y que no ha sido filtrada, interpretada o evaluada por alguien más. Son producto de una investigación o de una actividad eminentemente creativa. (12)

Por otro lado, las fuentes secundarias son el material que contiene información de carácter primario, pero que ha sido sintetizada, reorganizada, resumida

A este respecto también, Silvestrini (2008) nos dice que las fuentes secundarias, están especialmente diseñadas, para facilitar y maximizar el acceso a las fuentes primarias o a sus contenidos. (12) 


\section{RESULTADOS}

\section{LINEAS CONCEPTUALES}

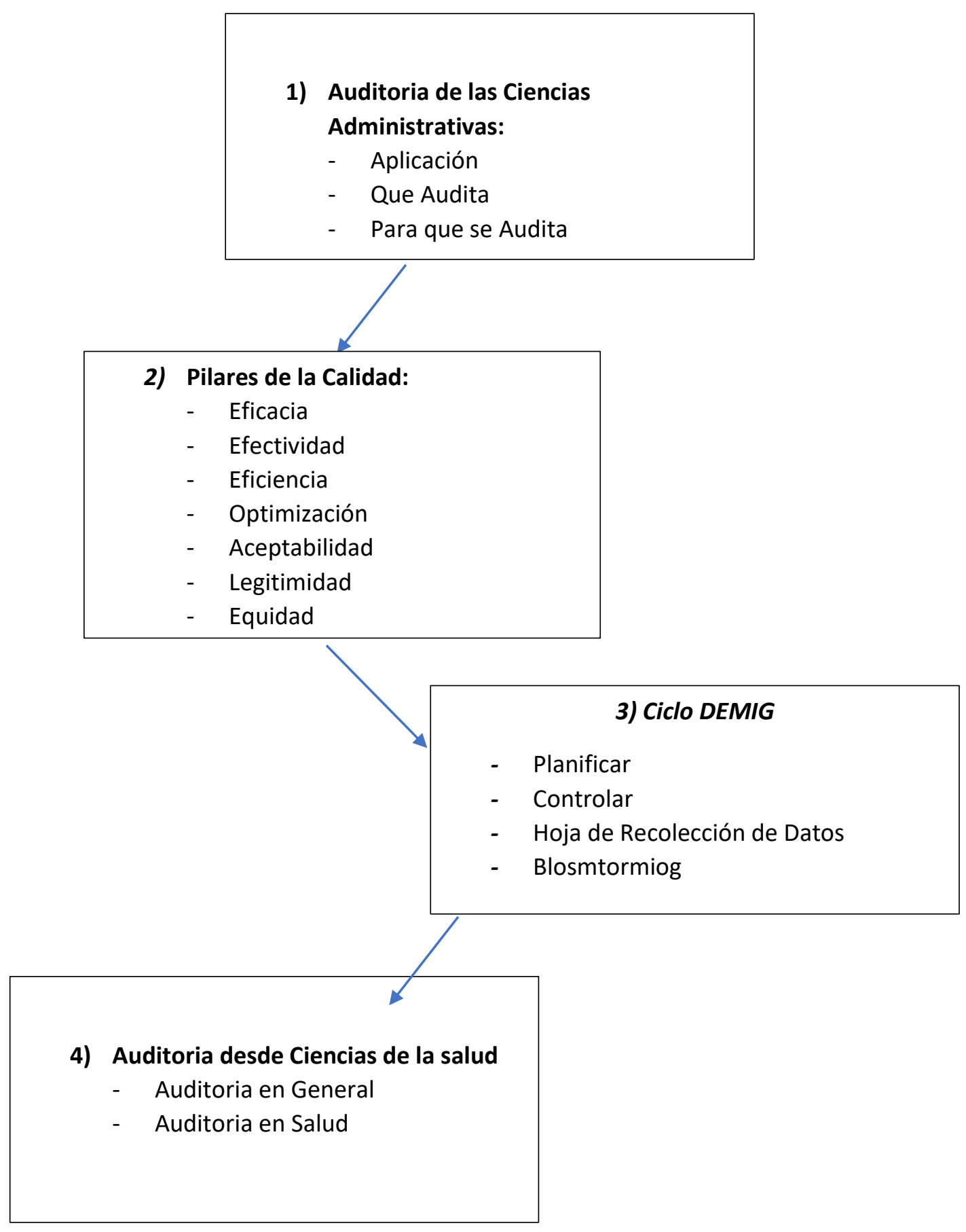

FUENTE: Autoría propia. 


\section{DISCUSIÓN}

En la presente Discusión de resultados hablaremos de 6 aspectos importantes abordados en la Monografía:

- La Auditoria como proceso de control en su etimología: en la presente monografía se realiza una revisión del término auditoría, los aspectos relacionados a los pilares de la calidad, el ciclo Deming y la relación que tiene con las ciencias administrativas. También aborda los tipos de auditoría: interna, independiente y gubernamental, su función e importancia. Se aborda el concepto de auditoría en salud, ya que enfocándose en su perspectiva administrativa y financiera.

- La Evaluación de la calidad definido por Donadevian Avedis: en la presente monografía se menciona la importancia de un cambio en el que se incluya un aumento de la equidad en la prestación del servicio, la eficiencia de la gestión y la efectividad de las actuaciones, para así lograr la satisfacción de las necesidades en la población de igual manera se realiza una revisión bibliográfica que aborda el concepto de calidad en salud de Avedis Donavedian.

- Situación actual de la Auditoria en salud en Colombia: en la presente monografía se hace un énfasis en indagar acerca de la situación actual de la salud en Colombia desde diferentes perspectivas comenzando por la del ministerio de salud y protección social, por otro lado la investigación apunta hacia el usuario y su grado de satisfacción, mencionando que en la mayoría de estos programas lo más importante es la calidad en la atención y el grado de humanización, puesto que apuntan más hacia los costos y la productividad.

- La Auditoria médica en su importancia para el sector salud: se aborda también en el presente estudia a la auditoría como una oportunidad para educarse y prevenir el error médico y no como una instancia sancionatoria y punitiva. Motivo por el cual la auditoría médica se convierte en un mecanismo que contribuye a evitar el riesgo de errores en el proceso de atención y de esta forma garantizar la calidad de la atención médico- asistencial.

- La Auditoria de calidad a diferencia de la auditoria tradicional: se consideró en la presente monografía dar una gran relevancia a la auditoría del servicio. La 
cual se enfoca en la atención que se le ofrece a los usuarios y el grado de satisfacción que manifiestan en relación con los principios de la atención en salud.

- La Importancia de la Auditoria en salud: es importante que se establezca el hábito de aplicarla en las autoevaluaciones cuando se aplican las auditorias en salud y no solo cuando se lleven a cabo las auditorías externas. igualmente se menciona que el proceso de auditoría en salud se debe realizar siempre con los registros clínicos, pues lo que se consigna en ellos es la evidencia de lo que se realizó y lo que se omitió revela lo que el profesional no ejecutó.

\section{CONCLUSIONES Y RECOMENDACIONES:}

Mediante el presente estudio se identifico que la auditoria es el proceso que busca el mejoramiento de la calidad para la prestación en servicios de la Salud. Consecuentemente se require tanto en su aplicación como en su contexto aplicarla desde la direccion teórica como práctica.asimismo se hace evidente el impacto que puede tener el desarrollo de la auditoria en los resultados de un procedimiento para un usuario. $Y$ práctica, con respecto a la inclusión de la auditoría dentro de la totalidad de procedimientos que presta una institución de salud, claramente basada en un marco de calidad y mejoramiento continuo.

Se entiende en ese sentido que la auditoria tiene un impacto no sólo en el desarrollo de una auditoría objetiva y de calidad, sino también en la percepción del rol del auditor y sus funciones, se hace necesario realizar estudios que permitan valorar de manera más profunda e imparcial la percepción que se tiene del profesional en auditoría, con el fin de desarrollar programas que generen una conciencia positiva no solo frente a la labor de la auditoría dentro de una institución, sino frente a quienes la llevan a cabo. 


\section{BIBLIOGRAFÍA}

1. Nacional Rdc. Ley 100 Bogotá; 1993.

2. Saludcoop. Revista semana. [Online].; 2018. Available from: https://www.semana.com/nacion/articulo/saludcoop-caida-imperio/239794-3.

3. Callao Hdsj. Manual de auditoria en salud. [Online].; 2004. Available from: http://www.hsj.gob.pe/web1/UGC/html/comite_auditoria/publicaciones/manual_au ditoria.pdf.

4. Hospitalaria MdIPSPdAalRdSACdG. Pautas de Auditoría para el Mejoramiento de la calidad de la atencion en salud. 2007;: p. 17,18,57.

5. Leonard WP. La Auditoría desde las Ciencias Administrativas, Evaluación de los Métodos. $\quad$ [Online].; $1991 . \quad$ Available from: https://educativopracticas.files.wordpress.com/2014/05/enfoque-de-leonardclase-1.pdf.

6. Avedia d. Los 7 pilares de la calidad, según Donabedian Avedis. [Online].; 1986. Available from: https://www.coursehero.com/file/p42bdopq/2221-Los-SietePilares-de-la-Calidad-De-acuerdo-a-un-art\%C3\%ADculo-escrito-por-el/.

7. Papurello N. Herramienta de control y mejoramiento de la calidad. [Online]. Available from: https://www.google.com/search?source=hp\&ei=LN4OXLgh6YPnAsKbhvAE\&q=H erramientas+de+Control++y+Mejoramiento+de+la+Calidad\&btnK=Buscar+con+G oogle\&oq=Herramientas+de+Control++y+Mejoramiento+de+la+Calidad\&gs_l=ps y-ab.3.33i22i29i30l7.17311.41148.42083.3.0.

8. Laverde MLGmP. Auditoria en Salud: Panamerica; 2003.

9. Ministerio de Salud y Protección Social. Informe del congreso de la república. [Online].; $2015 . \quad$ Available from: https://www.minsalud.gov.co/sites/rid/Lists/BibliotecaDigital/RIDE/DE/PES/inform e-congreso2015-2016.pdf.

10. Ministerio de Salud y Protección Social. Programa de apoyo a la reforma de salud, asociación centro de gestión hospitalaria 2007. [Online].; 2006.

11. Meyer. DBVDyWJ. LA INVESTIGACIÓN DESCRIPTIVA. [Online].; 2006. Available from: https://noemagico.blogia.com/2006/091301-la-investigaci-n-descriptiva.php.

12. María Silvestrini Ruiz MJVJ. FUENTES DE INFORMACIÓN PRIMARIAS, SECUNDARIAS Y TERCIARIAS. [Online].; 2008. Available from: http://ponce.inter.edu/cai/manuales/FUENTES-PRIMARIA.pdf. 